Internationale Innovationsdynamik, Spezialisierung und Wirtschaftswachstum in der EU

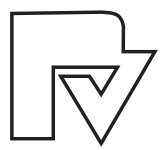




\section{Wirtschaftswissenschaftliche Beiträge}

\section{Informationen über die Bände 1-112 sendet Ihnen auf Anfrage gerne der Verlag.}

Band 113: B. Wieland, Telekommunikation und vertikale Integration, 1995.

ISBN 3-7908-0849-0

Band 114: D. Lucke, Monetäre Strategien zur Stabilisierung der Weltwirtschaft, 1995. ISBN 3-7908-0856-3

Band 115: F. Merz, DAX-Future-Arbitrage, 1995. ISBN 3-7908-0859-8

Band 116: T. Köpke, Die Optionsbewertung an der Deutschen Terminbörse, 1995. ISBN 3-7908-0870-9

Band 117: F. Heinemann, Rationalisierbare Erwartungen, 1995. ISBN 3-7908-0888-1

Band 118: J. Windsperger, Transaktionskostenansatz der Entstehung der Unternehmensorganisation, 1996. ISBN 3-7908-0891-1

Band 119: M. Carlberg, Deutsche Vereinigung. Kapitalbildung und Beschäftigung, 1996. ISBN 3-7908-0896-2

Band 120: U. Rolf, Fiskalpolitik in der Europäischen Währungsunion, 1996. ISBN 3-7908-0898-9

Band 121: M. Pfaffermayr, Direktinvestitionen im Ausland, 1996. ISBN 3-7908-0908-X

Band 122: A. Lindner, Ausbildungsinvestitionen in einfachen gesamtwirtschaftlichen Modellen, 1996. ISBN 3-7908-0912-8

Band 123: H. Behrendt, Wirkungsanalyse von Technologie und Gründerzentren in Westdeutschland, 1996. ISBN 3-7908-0918-7

Band 124: R. Neck (Hrsg.) Wirtschaftswissenschaftliche Forschung für die neunziger Jahre, 1996.

ISBN 3-7908-0919-5

Band 125: G. Bol, G. Nakhaeizadeh/

K.-H. Vollmer (Hrsg.) Finanzmarktanalyse und -prognose mit innovativen quantitativen Verfahren, 1996. ISBN 3-7908-0925-X

Band 126: R. Eisenberger, Ein Kapitalmarktmodell unter Ambiguität, 1996. ISBN 3-7908-0937-3

Band 127: M. J. Thenrillat, Der Schweizer Aktienmarkt, 1996. ISBN 3-7908-0941-1

Band 128: T. Lauer, Die Dynamik von Konsumgütermärkten, 1996. ISBN 3-7908-0948-9

Band 129: M. Wendel, Spieler oder Spekulanten, 1996. ISBN 3-7908-0950-0

Band 130: R. Olliges, Abbildung von Diffusionsprozessen, 1996. ISBN 3-7908-0954-3

Band 131: B. Wilmes, Deutschland und Japan im globalen Wettbewerb, 1996. ISBN 3-7908-0961-6 Band 132: A. Sell, Finanzwirtschaftliche Aspekte der Inflation, 1997. ISBN 3-7908-0973-X

Band 133: M. Streich, Internationale Werbeplanung, 1997. ISBN 3-7908-0980-2
Band 134: K. Edel, K.-A. Schäffer, W. Stier (Hrsg.) Analyse saisonaler Zeitreihen, 1997.

ISBN 3-7908-0981-0

Band 135: B. Heer, Umwelt, Bevölkerungsdruck und Wirtschaftswachstum in den Entwicklungsländern, 1997. ISBN 3-7908-0987-X

Band 136: Th. Christiaans, Learning by Doing in offenen Volkswirtschaften, 1997.

ISBN 3-7908-0990-X

Band 137: A. Wagener, Internationaler Steuerwettbewerb mit Kapitalsteuern, 1997.

ISBN 3-7908-0993-4

Band 138: P. Zweifel et al., Elektrizitätstarife und Stromverbrauch im Haushalt, 1997.

ISBN 3-7908-0994-2

Band 139: M. Wildi, Schätzung, Diagnose und Prognose nicht-linearer SETAR-Modelle, 1997. ISBN 3-7908-1006-1

Band 140: M. Braun, Bid-Ask-Spreads von Aktienoptionen, 1997. ISBN 3-7908-1008-8

Band 141: M. Snelting, Übergangsgerechtigkeit beim Abbau von Steuervergünstigungen und Subventionen, 1997. ISBN 3-7908-1013-4

Band 142: Ph. C. Rother, Geldnachfragetheoretische Implikationen der Europäischen Währungsunion, 1997. ISBN 3-7908-1014-2

Band 143: E. Steurer, Ökonometrische Methoden und maschinelle Lernverfahren zur Wechselkursprognose, 1997. ISBN 3-7908-1016-9

Band 144: A. Groebel, Strukturelle Entwicklungsmuster in Markt- und Planwirtschaften, 1997.

ISBN 3-7908-1017-7

Band 145: Th. Trauth, Innovation und Außenhandel, 1997. ISBN 3-7908-1019-3

Band 146: E. Lübke, Ersparnis und wirtschaftliche Entwicklung bei alternder Bevölkerung, 1997. ISBN 3-7908-1022-3

Band 147: F. Deser, Chaos und Ordnung im Unternehmen, 1997. ISBN 3-7908-1023-1

Band 148: J. Henkel, Standorte, Nachfrageexternalitäten und Preisankündigungen, 1997.

ISBN 3-7908-1029-0

Band 149: R. Fenge, Effizienz der Alterssicherung, 1997. ISBN 3-7908-1036-3

Band 150: C. Graack, Telekommunikationswirtschaft in der Europäischen Union, 1997. ISBN 3-7908-1037-1

Band 151: C. Muth, Währungsdesintegration Das Ende von Währungsunionen, 1997. ISBN 3-7908-1039-8

Band 152: H. Schmidt, Konvergenz wachsender Volkswirtschaften, 1997. ISBN 3-7908-1055-X

Fortsetzung auf Seite 537 
Andre Jungmittag

\section{Internationale Innovationsdynamik, Spezialisierung und Wirtschaftswachstum in der EU}

Mit 78 Abbildungen und 69 Tabellen

$\underline{\text { Physica-Verlag }}$

Ein Unternehmen von Springer 


\section{Reihenherausgeber}

Werner A. Müller

\section{Autor}

PD Dr. Andre Jungmittag

Bergische Universität Wuppertal

FB B Wirtschafts- und Sozialwissenschaften

Lehrstuhl für Makroökonomische Theorie und Politik

Gaußstraße 20

42119 Wuppertal

E-mail: jungmittag@wiwi.uni-wuppertal.de

ISSN 1431-2034

ISBN 3-7908-1713-9 Physica-Verlag Heidelberg

Bibliografische Information Der Deutschen Bibliothek

Die Deutsche Bibliothek verzeichnet diese Publikation in der Deutschen Nationalbibliografie; detaillierte bibliografische Daten sind im Internet über $<$ http://dnb.ddb.de $>$ abrufbar.

Dieses Werk ist urheberrechtlich geschützt. Die dadurch begründeten Rechte, insbesondere die der Übersetzung, des Nachdrucks, des Vortrags, der Entnahme von Abbildungen und Tabellen, der Funksendung, der Mikroverfilmung oder der Vervielfältigung auf anderen Wegen und der Speicherung in Datenverarbeitungsanlagen, bleiben, auch bei nur auszugsweiser Verwertung, vorbehalten. Eine Vervielfältigung dieses Werkes oder von Teilen dieses Werkes ist auch im Einzelfall nur in den Grenzen der gesetzlichen Bestimmungen des Urheberrechtsgesetzes der Bundesrepublik Deutschland vom 9. September 1965 in der jeweils geltenden Fassung zulässig. Sie ist grundsätzlich vergütungspflichtig. Zuwiderhandlungen unterliegen den Strafbestimmungen des Urheberrechtsgesetzes.

Physica-Verlag Heidelberg

ein Unternehmen von Springer Science+Business Media GmbH

springer.de

(C) Physica-Verlag Heidelberg 2006

Printed in Germany

Die Wiedergabe von Gebrauchsnamen, Handelsnamen, Warenbezeichnungen usw. in diesem Werk berechtigt auch ohne besondere Kennzeichnung nicht zu der Annahme, dass solche Namen im Sinne der Warenzeichenund Markenschutz-Gesetzgebung als frei zu betrachten wären und daher von jedermann benutzt werden dürften.

Umschlaggestaltung: Erich Kirchner, Heidelberg

Herstellung: LE-T $\mathrm{E}$ X Jelonek, Schmidt \& Vöckler GbR Leipzig

SPIN $11688150 \quad 88 / 3100-543210$ - Gedruckt auf säurefreiem und alterungsbeständigem Papier 


\section{Vorwort}

$\mathrm{Zu}$ Beginn des 21. Jahrhunderts sind die positiven Auswirkungen von technischem Fortschritt und Innovationen auf das Wirtschaftswachstum allgemein anerkannt. Dennoch stellen die Mechanismen, die Innovationen in weitreichende ökonomische Effekte übersetzen, noch ein breites Terrain mit vielen offenen Forschungsfragen dar. So dürfte neben der allgemeinen Innovationsfähigkeit eines Landes auch seine technologische und wirtschaftliche Spezialisierung die gesamtwirtschaftliche Leistungsfähigkeit beeinflussen. Zudem können technologisch und wirtschaftlich zurückliegende Länder durch die Imitation von Technologien anderer Länder aufholen. Es ist daher das Ziel dieser Studie, die Zusammenhänge zwischen der Innovationsdynamik von Volkswirtschaften, ihren technologischen und wirtschaftlichen Spezialisierungen sowie ihren Wirtschaftswachstums- und Produktivitätsentwicklungen theoretisch und empirisch zu untersuchen. Damit solch eine Analyse hinreichend Erklärungskraft erhält, beschränkt sie sich auf die relativ homogene Gruppe der EU15-Staaten.

Die vorliegende Arbeit wurde im Juli 2005 als Habilitationsschrift vom Fachbereich Wirtschafts- und Sozialwissenschaften der Bergischen Universität Wuppertal angenommen. Für die Betreuung der Arbeit, die mit zahlreichen wertvollen Diskussionen und Anregungen verbunden war, bin ich Herrn Prof. Dr. Paul J.J. Welfens, Lehrstuhl für Makroökonomische Theorie und Politik an der Bergischen Universität Wuppertal, zu großem Dank verpflichtet. Zudem danke ich Herrn Prof. Dr. Gerhard Arminger, Lehrstuhl für Wirtschaftsstatistik an der Bergischen Universität Wuppertal, und Herrn Prof. Dr. Hans Gerhard Strohe, Lehrstuhl für Statistik und Ökonometrie an der Universität Potsdam, für die Übernahme der weiteren Gutachten.

Im Laufe der Erstellung der Arbeit erhielt ich von verschiedenen Seiten weitere Unterstützung. Teile des dritten Kapitels entstanden während eines Forschungsaufenthalts im Herbst 2001 am German-American Center for Visiting Scholars (GACVS) der Alexander von Humboldt Stiftung und am American Institute for Contemporary German Studies (AICGS)/The Johns Hopkins University in Washington D.C. Beiden Institutionen danke ich für die Bereitstellung einer anregenden und produktiven wissenschaftliche Umgebung. Teile des fünften Kapitels wurden während meines Aufenthalts als Gastwissenschaftler bei der DG II Wirtschaft und Finanzen der Europäischen Kommission im Herbst 2003 grundlegend erstellt und dort auch in einem Seminar präsentiert. Auch hier möchte ich mich für die stimulierende Forschungsatmosphäre erkenntlich zeigen und insbesondere 
Herrn Dr. Werner Röger und Herrn Prof. Dr. Klaus Wälde für ihre hilfreichen Hinweise und Kommentare danken. Weiterhin bedanke ich mich bei Herrn Prof. Dr. Jürgen Wolters, dessen hilfreiche Anregungen auf einem Workshop zu einer Sonderausgabe der Zeitschrift "International Economics and Economic Policy" im Februar $2004 \mathrm{zu}$ einer weiteren Verbesserung dieses Teils der Arbeit führten.

Zudem bedanke ich mich bei meinen Kollegen und Kolleginnen Frau DiplomVolkswirtin Dora Borbély, Herrn Diplom-Volkswirt Albrecht Kauffmann, Herrn Diplom-Ökonom Martin Keim, Herrn Diplom-Volkswirt Christopher Schumann und Herrn Dr. Ralf Wiegert am Lehrstuhl für Wirtschaftspolitik/Internationale Wirtschaftsbeziehungen an der Universität Potsdam sowie am Lehrstuhl für Makroökonomische Theorie und Politik an der Bergischen Universität Wuppertal für ihre umfassende Unterstützung während der gesamten Habilitationsphase. Die angenehme Arbeitsatmosphäre an beiden Lehrstühlen hat sicher auch einen Beitrag zum Gelingen dieser Untersuchung geleistet.

Vielfältigen Rückhalt habe ich durch meine Frau Gudrun Pohl erfahren. Ihr verdanke ich wertvolle Anregungen, großen Zuspruch und viel Verständnis während der ganzen Zeit. Nicht zuletzt hat sie als Nichtökonomin die Last auf sich genommen, die gesamte Arbeit Korrektur zu lesen.

Wuppertal, im Januar 2006

Andre Jungmittag 


\section{Inhaltsverzeichnis}

1 Einführung 1

2 Innovationsdynamik, Spezialisierung und Wirtschaftswachstum: Theoretische Erklärungsansätze

2.1 Charakter des technischen Fortschritts im neoklassischen Wachstumsmodell . . . . . . . . . . . . . . . . . 12

2.2 Exportbasiertes Wachstum, Spezialisierung und kumulative Verursachung ..................... . . 17

2.2.1 Das Grundmodell . . . . . . . . . . . . . . . . . . 19

2.2.2 Die Berücksichtigung einer Zahlungsbilanzrestriktion . . . 23

2.2.3 Technologische Leistungsfähigkeit und die Modellierung der Entwicklung sektoraler Spezialisierungsmuster . . . . . . . 25

2.3 Technologielücke, Produktzyklus und Spezialisierung . . . . . . . 34

2.3.1 Technologische Lücke und wirtschaftliche Aufholprozesse . 35

2.3.2 Technologische Lücke, Außenhandel und Produktzyklus . . 52

2.4 Evolutorische Ökonomik . . . . . . . . . . . . . . . . . 66

2.4.1 Grundlagen der evolutorischen Ökonomik . . . . . . . . . . 67

2.4.2 Innovationen und Spezialisierung in evolutorischen Wachstumsmodellen .................. . 71

2.4.3 Das Konzept der nationalen Innovationssysteme und langfristiges Wirtschaftswachstum . . . . . . . . . . 88

2.5 Neue Wachstumstheorie mit endogenem technischen Fortschritt . 94

2.5.1 Learning-by-Doing als Triebkraft endogenen Wachstums . 95

2.5.1.1 Die geschlossene Volkswirtschaft . . . . . . . . 95

2.5.1.2 Die offene Volkswirtschaft . . . . . . . . . . . . . 104

2.5.2 FuE als Triebkraft endogenen Wachstums . . . . . . . . . 118

2.5.2.1 Die geschlossene Volkswirtschaft . . . . . . . 119

2.5.2.2 Die offene Volkswirtschaft . . . . . . . . . . . 152

2.6 Zusammenfassung und Ausblick . . . . . . . . . . . . . . . 162

Anhang: Das neoklassische Wachstumsmodell mit exogenem technischen

Fortschritt . . . . . . . . . . . . . . . . 169

3 Innovationsdynamik in der EU: Konvergenz oder Divergenz? 201

3.1 Nationale Innovationsfähigkeit: Begriffsdefinition und Meßkonzept 202 
3.2 Langfristige Trends des Innovationsoutputs . . . . . . . . . . . . 206

3.3 Ansätze zur Konvergenzmessung . . . . . . . . . . . . . . . . . . 216

3.3.1 Querschnittstests der Konvergenzhypothese . . . . . . . . 219

3.3.2 Zeitreihen- und Paneldatentests der Konvergenzhypothese 225

3.4 Ergebnisse der empirischen Analyse . . . . . . . . . . . . . . . 235

3.4.1 Tests auf $\sigma$-Konvergenz . . . . . . . . . . . . . . 235

3.4.2 Tests auf $\beta$-Konvergenz . . . . . . . . . . . . . . . . 240

3.4.3 Zeitreihen- und Paneldatentests der Konvergenzhypothese 249

3.5 Zusammenfassung . . . . . . . . . . . . . . . . . . . . 270

4 Die Entwicklung der technologischen und wirtschaftlichen Spezialisierungen der EU-Staaten 273

4.1 Methodik und Datengrundlage . . . . . . . . . . . . . . . . 275

4.1.1 Absolute Spezialisierungsmaße . . . . . . . . . . . . . . 275

4.1.2 Relative Spezialisierungsmaße . . . . . . . . . . . . . . 279

4.1.3 Überprüfung auf $\beta$ - und $\sigma$-Spezialisierung . . . . . . . . . 282

4.1.4 Die Datenbasis . . . . . . . . . . . . . . . . . . 285

4.2 Die Entwicklung der Smithianischen Spezialisierungen . . . . . . . 289

4.2.1 Technologische Spezialisierung . . . . . . . . . . . . . . . 289

4.2.2 Produktionsspezialisierung . . . . . . . . . . . . . . . 313

4.2.3 Exportspezialisierung . . . . . . . . . . . . . . . . . . 325

4.2.4 Außenhandelsspezialisierung . . . . . . . . . . . . . . 337

4.3 Die Entwicklung der Ricardianischen Spezialisierungen . . . . . . 344

4.3.1 Technologische Spezialisierung . . . . . . . . . . . . . . 345

4.3.2 Produktionsspezialisierung . . . . . . . . . . . . . . 367

4.3.3 Exportspezialisierung . . . . . . . . . . . . . . . . . 380

4.3.4 Außenhandelsspezialisierung . . . . . . . . . . . . . . . 391

4.4 Zusammenfassung . . . . . . . . . . . . . . . . . . . . . . . 401

5 Der Beitrag von Innovationen und Spezialisierung zu Wirtschaftswachstum und Konvergenz in der EU

5.1 Konvergenz der Pro-Kopf-Einkommen und Arbeitsproduktivitäten in der EU? . . . . . . . . . . . . . . . . . . . . . 416

5.1.1 Ergebnisse bisheriger empirischer Studien . . . . . . . . . 416

5.1.2 Ergebnisse der Tests auf $\sigma$ - und $\beta$-Konvergenz . . . . . . . 427

5.1.3 Ergebnisse der Zeitreihen- und Paneldatentests der Konvergenzhypothese . . . . . . . . . . . . . . . . . 432

5.2 Innovationen und technologische Spezialisierung als Triebkräfte von Wirtschaftswachstum und Konvergenz in der EU . . . . . . . 439

5.2.1 Methodik und Datengrundlage . . . . . . . . . . . . . . . . 440

5.2.1.1 Das empirische Modell . . . . . . . . . . . . . . . 441

5.2.1.2 Dekomposition des Wirtschaftswachstums und der Konvergenzmaße . . . . . . . . . . . . . . 443

5.2.1.3 Datengrundlage und „Pre-Testing” . . . . . . . . 445

5.2.2 Ergebnisse der empirischen Analyse . . . . . . . . . . . . . 453

5.2.2.1 Schätzergebnisse . . . . . . . . . . . . . . 453 
5.2.2.2 Ergebnisse der Wachstumsdekompositionen . . 459

5.2.2.3 Ergebnisse der Konvergenzdekompositionen ... 464

5.3 Technologische Spezialisierung als Triebkraft der wirtschaftlichen Spezialisierung . . . . . . . . . . . . . . . 4 470

5.3.1 Theoretischer Ansatz und empirische Modellierung . . . . 471

5.3.2 Ergebnisse der empirischen Analyse . . . . . . . . . . . . 477

5.4 Zusammenfassung . . . . . . . . . . . . . . . . . 488

$\begin{array}{lll}6 & \text { Schlußbetrachtung } & 491\end{array}$

$\begin{array}{ll}\text { Literaturverzeichnis } & 503\end{array}$

$\begin{array}{ll}\text { Tabellenverzeichnis } & 529\end{array}$

$\begin{array}{ll}\text { Abbildungsverzeichnis } & 533\end{array}$ 